\title{
The dollars and sense of drug-eluting stents
}

\author{
James M. Brophy
}

ß S See related articles pages 323, 345

$\mathrm{P}$ ercutaneous transluminal coronary angioplasty, now known as percutaneous coronary intervention (PCI), was described for the first time 26 years ago ${ }^{1}$ and has undergone many technological advances, most notably the introduction of endovascular metallic scaffolding, more commonly referred to as coronary stents. PCI is now the most widely used cardiovascular revascularization procedure, with about 35000 procedures performed annually in Canada and over 750000 in the United States. Stenting has become widespread over the last 10 years and has resulted in lower rates of restenosis, the Achilles heal of angioplasty. ${ }^{2}$ In an attempt to further reduce restenosis rates, drug-eluting stents have recently been added to the therapeutic armamentarium.

In this issue, Shrive and colleagues ${ }^{3}$ have performed the valuable exercise of estimating the cost-effectiveness of this new technology using a pooled estimate of the relative reduction of restenosis at 9-12 months from 4 clinical trials of sirolimus-eluting stents. This medium-term efficacy estimate, combined with measures of resource utilization and quality of life (QOL) from the Alberta Provincial Project for Outcome Assessment in Coronary Heart Disease (APPROACH) database, were used to populate a decision analysis model to predict longer term expected benefit. Although efficacy estimates are best obtained from clinical trials, the authors are to be commended for estimating the other parameters from a real-world unselected population. For example, the frequency of repeat revascularization in this database was $8.2 \%$, whereas randomized controlled trials, with their compulsory protocol angiograms, suggest a 3 -fold higher rate. The avoidance of unrealistic parameter estimates is obviously essential for study validation.

Shrive and colleagues report a base case cost-utility ratio of \$58 721 per quality-adjusted life-year (QALY) gained with the use of sirolimus-eluting stents. Not surprisingly, when the risk of restenosis is higher (in elderly patients and in those with diabetes) the cost-effectiveness ratio falls, and conversely it may exceed $\$ 100000$ per QALY gained when the rates of restenosis are low. The authors conclude that the use of sirolimus-eluting stents has a cost-effectiveness profile similar to that of other accepted technologies, including the use of conventional stents versus simple balloon angioplasty in cases of acute myocardial infarction $(\$ 65000$ per QALY gained). ${ }^{4}$ American investigators ${ }^{5}$ have reported a numerically similar incremental cost-effectiveness ratio for the use of sirolimus-eluting stents (US\$27 540), but the present study offers the advantages of local data collection and, being free of direct corporate sponsorship, perhaps a less biased estimate. The conclusions of both studies ${ }^{3,5}$ are limited to sirolimus-eluting stents and do not apply to other stent models. ${ }^{6}$ Definitive conclusions regarding the relative efficacy and cost-effectiveness of competing stents must await data from direct head-to-head trials.

\section{Are there study limitations?}

Although most assumptions in the model studied by Shrive and colleagues appear reasonable, including a possible reduction in coronary artery bypass grafting (CABG) as a repeat procedure, it must be noted that the trials comparing coated stents with bare-metal stents have not shown any statistically significant reductions in rates of CABG, myocardial infarction or death.

Also, as many as $40 \%$ of the repeat revascularizations in the first year may not have been attributed to clinical restenosis but, rather, to disease progression. ${ }^{7}$ In subsequent years disease progression is 4 times more likely than stent restenosis to be responsible for adverse clinical outcomes (hazard ratio 6.3\% v. 1.7\%). ${ }^{7}$ If these observations hold in the Canadian context, the expected long-term benefits of drug-eluting stents may be substantially mitigated.

The accuracy of the QOL measurements is also fundamental to the overall assessment of the benefit of sirolimuseluting stents. Although a major advantage of the study by Shrive and colleagues is their local data collection, only $27 \%$ of eligible candidates were sampled, and it is unclear if any selection bias was present, when the measurements were obtained and what their variability was. In addition, QOL measurement errors may have existed, as highlighted by the lack of differences between repeat revascularizations by PCI or CABG. Moreover, another study ${ }^{4}$ showing similar absolute reduction in repeat revascularizations and using the same QOL metric reported only one-sixth the improvement of that reported by Shrive and colleagues. Because the symptoms of restenosis are generally ephemeral, one would not expect to have major differences in an annual QOL metric.

Given these difficulties in assessing QOL, benefits of drug-eluting stents have been alternatively expressed in dollars per revascularization avoided, with estimates ranging from Can $\$ 7200^{8}$ and US\$1650..$^{5}$ These costs represent an additional premium beyond any savings associated with reduced repeat revascularizations. 


\section{Are drug-eluting stents good value for the money?}

This value judgement is exceedingly difficult to make, even with the contribution of the present study. American patients have expressed a willingness to pay US\$273 to reduce restenosis rates by amounts offered by sirolimus-eluting stents, ${ }^{9}$ although wealthier patients were willing to pay more. Data from Canadian patients are unavailable. Nevertheless, there seems to be a "disconnect" between what patients, and possibly administrators, feel this technology is worth and current pricing. Before implementation of this new technology becomes a priority, more discussion will be required, not only by the cardiovascular community but also by the medical community and general population.

The total impact of sirolimus-eluting stents on our health care budget, even after allowing for savings from a reduction in repeat revascularizations, may approach $\$ 35$ million in Quebec alone ${ }^{8}$ and, by extrapolation, $\$ 75$ million in Canada. Thus, this single technology could consume close to $4 \%$ of the $\$ 2.1$ billion of new funding for the current fiscal year recently negotiated between the federal and provincial governments. Our present evidence base appears inadequate for this level of commitment, since the majority of trials have looked at relatively low-risk patients and it is unclear whether high-risk patients will experience the same benefits. There also remains a paucity of data regarding long-term effects. ${ }^{10,11}$ Are the benefits maintained, or is restenosis merely delayed? About $90 \%$ of patients do not experience clinical restenosis with conventional stents and therefore would not derive any additional benefit from having a sirolimus-eluting stent. Health care budgets are necessarily limited, and investment in this technology may have to come from other health care sectors, the so-called opportunity cost.

How should practising clinicians interpret the data reported by Shrive and colleagues? Our current infatuation with interventional cardiology must be questioned, since both old $^{12}$ and recent ${ }^{13}$ studies involving stable patients have failed to show a reduction in either mortality or myocardial infarction with PCI compared with medical treatment. QOL is improved in active patients, but for many this is not a major issue. In addition to recognizing the inevitable scarcity of our resources, clinicians must temper the natural enthusiasm of interventional researchers and think of the overall health of their patients by considering, for example, an alternative in- creased investment in more basic primary and secondary prevention and treatment programs. Studies such as the one by Shrive and colleagues will assist us in navigating the challenging chasm between clinical Scylla and financial Charybdis.

James Brophy is with the Division of Cardiology and Clinical Epidemiology, McGill University Health Centre Technology Assessment Unit, Royal Victoria Hospital, Montréal, Que.

Competing interests: None declared.

\section{References}

1. Gruntzig AR, Senning A, Siegenthaler WE. Nonoperative dilatation of coronary-artery stenosis: percutaneous transluminal coronary angioplasty. $N$ Engl 7 Med 1979;301:61-8.

2. Brophy JM, Belisle P, Joseph L. Evidence for use of coronary stents. A hierarchical bayesian meta-analysis. Ann Intern Med 2003;138:777-86.

3. Shrive FM, Manns BJ, Galbraith PD, Knudtson ML, Ghali WA; for the APPROACH Investigators. Economic evaluation of sirolimus-eluting stents. CMA7 2005;172(3):345-51.

4. Cohen DJ, Taira DA, Berezin R, Cox DA, Morice MC, Stone GW, et al Cost-effectiveness of coronary stenting in acute myocardial infarction: results from the Stent Primary Angioplasty in Myocardial Infarction (stent-PAMI) trial. Circulation 2001;104(25):3039-45.

5. Cohen DJ, Bakhai A, Shi C, Githiora L, Lavelle T, Berezin RH, et al; SIRIUS Investigators. Cost-effectiveness of sirolimus-eluting stents for treatment of complex coronary stenoses: results from the Sirolimus-Eluting Balloon Expandable Stent in the Treatment of Patients With De Novo Native Coronary Artery Lesions (SIRIUS) trial. Circulation 2004;110(5):508-14.

6. Babapulle MN, Joseph L, Belisle P, Brophy JM, Eisenberg MJ. A hierarchical Bayesian meta-analysis of randomised clinical trials of drug-eluting stents. Lancet 2004;364:583-91.

7. Cutlip DE, Chhabra AG, Baim DS, Chauhan MS, Marulkar S, Massaro J, et al. Beyond restenosis: five-year clinical outcomes from second-generation coronary stent trials. Circulation 2004;110(10):1226-30.

8. Brophy J, Erickson L, for Agence d'Evaluation des Technologies et des Modes d'Interventions en Santé (AETMIS). An economic analysis of drug eluting coronary stents: a Québec perspective. Québec: AETMIS; 2004.

9. Greenberg D, Bakhai A, Neumann PJ, Cohen DJ. Willingness to pay for avoiding coronary restenosis and repeat revascularization: results from a contingent valuation study. Health Policy 2004;70:207-16.

10. McFadden EP, Stabile E, Regar E, Cheneau E, Ong AT, Kinnaird T, et al Late thrombosis in drug-eluting coronary stents after discontinuation of antiplatelet therapy. Lancet 2004;364(9444):1519-21.

11. Virmani R, Guagliumi G, Farb A, Musumeci G, Grieco N, Motta T, et al. Localized hypersensitivity and late coronary thrombosis secondary to a sirolimus-eluting stent: Should we be cautious? Circulation 2004;109(6):701-5.

12. Parisi AF, Folland ED, Hartigan P. A comparison of angioplasty with medical therapy in the treatment of single-vessel coronary artery disease. Veterans Affairs ACME Investigators. N Engl f Med 1992;326:10-6.

13. Hueb W, Soares PR, Gersh BJ, Cesar LA, Luz PL, Puig LB, et al. The medicine, angioplasty, or surgery study (MASS-II): a randomized, controlled clini$\mathrm{cal}$ trial of three therapeutic strategies for multivessel coronary artery disease: one-year results. 7 Am Coll Cardiol 2004;43(10):1743-51.

Correspondence to: Dr. James M. Brophy, Division of Cardiology and Clinical Epidemiology, McGill University Health Centre Technology Assessment Unit, Royal Victoria Hospital, Ross 4.12, 687 Pine Ave. W, Montréal QC H3A 1A1; fax 514 843-1493; james.brophy@mcgill.ca 